\title{
Diagnostic Value of FDG-PET/CT for the Identification of Extranodal Extension in Patients With Head and Neck Squamous Cell Carcinoma
}

\author{
RYO TOYA $^{1}$, TETSUO SAITO ${ }^{1}$, TOMOHIKO MATSUYAMA ${ }^{1}$, YUDAI KAI ${ }^{2,3}$, SHINYA SHIRAISHI $^{4}$, \\ DAIZO MURAKAMI ${ }^{5}$, RYOJI YOSHIDA ${ }^{6}$, TAKAHIRO WATAKABE ${ }^{1}$, FUMI SAKAMOTO $^{3}$, NORIKO TSUDA ${ }^{3}$, \\ HIDETAKA ARIMURA $^{3}$, YORIHISA ORITA ${ }^{5}$, HIDEKI NAKAYAMA ${ }^{6}$ and NATSUO OYA ${ }^{1}$ \\ ${ }^{1}$ Department of Radiation Oncology, Faculty of Life Sciences, Kumamoto University, Kumamoto, Japan; \\ ${ }^{2}$ Department of Radiological Technology, Kumamoto University Hospital, Kumamoto, Japan; \\ ${ }^{3}$ Department of Health Sciences, Graduate School of Medical Sciences, Kyushu University, Fukuoka, Japan; \\ ${ }^{4}$ Department of Diagnostic Radiology, Faculty of Life Sciences, Kumamoto University, Kumamoto, Japan; \\ ${ }^{5}$ Department of Otolaryngology-Head and Neck Surgery, \\ Faculty of Life Sciences, Kumamoto University, Kumamoto, Japan; \\ ${ }^{6}$ Department of Oral and Maxillofacial Surgery, Faculty of Life Sciences, Kumamoto University, Kumamoto, Japan
}

\begin{abstract}
Background/Aim: We evaluated the diagnostic value of functional imaging with $\left[{ }^{18} \mathrm{~F}\right]$-fluoro-2-deoxy-D-glucose (FDG)-positron emission tomography/computed tomography (PET/CT) for the identification of extranodal extension (ENE) in patients with head and neck squamous cell carcinoma (HNSCC). Patients and Methods: In this study, 94 patients with HNSCC who underwent FDG-PET/CT were enrolled. We recorded the maximum standardized uptake value $\left(S U V_{\max }\right)$, compared the results with pathologic findings, and evaluated the diagnostic performance of using a $S U V_{\max }$ cut-off value for ENE. Results: Of the 566 dissected levels examined, 53 (9.4\%) exhibited ENE. The mean $S U V_{\text {max }}$ of $L N$ with and without ENE were 6.67 and 1.64 , respectively $(p<0.001)$. A receiver operating characteristics (ROC) curve analysis for $S U V_{\max }$ showed an area under the ROC curve of 0.913. A SUV $V_{\max }$ cutoff of 3.0 achieved diagnostic performance for identifying ENE with sensitivity, specificity, and accuracy of $81.1 \%, 94.3 \%$ and 93.1\%, respectively. Conclusion: FDG-PET/CT findings using a $S U V_{\max }$ cut-off of 3.0 provides appropriate diagnostic value in identifying ENE.
\end{abstract}

This article is freely accessible online.

Correspondence to: Ryo Toya, Department of Radiation Oncology, Faculty of Life Sciences, Kumamoto University, 1-1-1 Honjo, Chuo-ku, Kumamoto 860-8556, Japan. Tel: +81 963735261, Fax: +81963624330, e-mail: ryo108@kumamoto-u.ac.jp

Key Words: Head and neck cancer, squamous cell carcinoma, positron emission tomography, extranodal extension, lymph node metastasis.
Intensity-modulated radiation therapy (IMRT) has become the standard technique for radiation treatment of head and neck squamous cell carcinoma (HNSCC). IMRT has been shown to reduce the dose distribution to critical organs at risk, which has led to a significant reduction in treatmentrelated morbidity, however, the rapid dose drop-off beyond the target volume makes treatment success highly dependent on the accurate determination of the target (1).

Extranodal extension (ENE), which occurs in approximately $60 \%$ of HNSCC patients with regional lymph node metastases (LNM), has a strongly negative impact on prognosis and outcome $(2,3)$. After the 2016 revision of the Union for International Cancer Control (UICC) TNM staging system, ENE has been included in nodal staging. Nodal clinical target volume (CTV) in HNSCC takes ENE into account during radiotherapy treatment planning. A 0-5 mm expansion of the nodal gross tumour volume (GTV) is typically proposed for CTV in patients with LNM without ENE (1). When LNM with ENE is present, a $10 \mathrm{~mm}$ nodal CTV margin around the GTV is recommended (4). Therefore, it is important to identify the existence of ENE before treatment.

Functional imaging with $\left[{ }^{18} \mathrm{~F}\right]$-fluoro-2-deoxy-D-glucose (FDG)-positron emission tomography (PET) provides information on glucose metabolism and assists management of HNSCC patients with respect to staging and prediction of treatment outcome $(5,6)$. However, the value of FDGPET in identifying ENE is not fully discussed (7-10). In this study, we evaluated whether FDG-PET provides diagnostic value for identification of ENE in patients with HNSCC. 


\section{Patients and Methods}

Patients. This retrospective study received institutional review board approval. Between April 2008 and March 2017, 94 patients with pathologically confirmed HNSCC who underwent FDG-PET/ computed tomography (CT) imaging in our hospital before surgery were included. Patients with a prior history of head and neck cancer, neck surgery, chemoradiotherapy, and those who underwent PET/CT imaging more than 6 weeks prior to the operative date were excluded $(9,11)$. Prior informed consent was obtained from all the patients for the use of their images in future studies. The primary cancer sites were oral cavity in 58 patients, oropharynx in 6 , hypopharynx in 20 , and larynx in 16. Six patients had cancer involving multiple sites in the head and neck. All patients underwent a conventional preoperative work up, including endoscopy, ultrasound, contrastenhanced CT, and magnetic resonance imaging (MRI), in addition to FDG-PET/CT imaging. The decision to perform selective or radical neck dissection (ND) was made by experienced head and neck surgeons, based on results of the diagnostic workup and patient performance status. ND specimens were removed en bloc and divided by nodal level; pathologic findings for the existence of LNM and ENE were recorded at each anatomic level (5).

FDG-PET/CT imaging. FDG-PET/CT images were obtained using a 3D PET/CT scanner (Gemini GXL 16; Philips Medical Systems, Cleveland, OH, USA). All patients fasted for 6 hours before the imaging procedure and underwent two routine whole-body PET/CT scans in a single session after receiving an injection of FDG (185$370 \mathrm{MBq}$ ): one at 60-90 min (early scan) and a second at 120-150 min (delayed scan). In the early scan, we acquired CT images [63 $\mathrm{mA}, 120 \mathrm{kV}, 512 \times 512$ matrix, 600-mm field of view (FOV), 5-mm slice thickness] and then performed emission measurements in $3 \mathrm{D}$ mode with a $144 \times 144$ matrix. The emission scan time per bed position was $2 \mathrm{~min}$; 10-12 bed positions (FOV $576 \mathrm{~mm}$ ) were acquired. Attenuation correction was with CT transmission data; emission images were reconstructed using the line of response-rowaction maximum likelihood algorithm. Reconstructed images had a $4 \mathrm{~mm}$ slice thickness. Emission scan time per bed position of the delayed scan was 3 min; 4-6 bed positions were acquired. Other imaging protocols were the same as those of the early scan (12).

FDG-PET/CT data and statistical analysis. One radiation oncologist with 15 years of experience in diagnosing and treating HNSCC reviewed PET and CT images of the early scan. The maximum standardized uptake value $\left(\mathrm{SUV}_{\max }\right)$ of a represented $\mathrm{LN}$ or the area of the highest SUV were recorded at each nodal level (I-V) and compared with pathologic findings of ENE based on the nodal level $(5,9)$.

The Mann-Whitney $U$-test was performed to evaluate the association of $\mathrm{SUV}_{\max }$, with ENE. Receiver operating characteristics (ROC) analysis with Youden index was performed to determine the best $\mathrm{SUV}_{\max }$ cut-off for the identification of ENE. Statistical calculations were performed with SPSS software, version 24.0 (IBM, Armonk, NY, USA). Differences with $p$-values of $<0.05$ were considered statistically significant.

\section{Results}

Of the 94 patients, $54(57.4 \%)$ patients had LNM. Of these, $23(24.4 \%)$ patients had LNM with ENE. The other 40 (42.6\%) patients were recorded as N0. Dissection of 566 nodal levels was performed; correlation between $\mathrm{SUV}_{\max }$ and histologic data was analyzed. ENE was present in 53 levels $(9.4 \%)$ of $106(18.7 \%)$ pathologically positive nodal levels. The mean $\mathrm{SUV}_{\text {max }}$ of all nodal levels was 2.11 2.44 . The mean $\mathrm{SUV}_{\max }$ of $\mathrm{LN}$ with and without ENE was $6.67 \pm 4.74($ range $=0.7-18.9)$ and $1.64 \pm 1.38($ range=0.5-23.4), respectively $(p<0.001$, Figure 1$)$.

The ROC curve analysis of $\mathrm{SUV}_{\max }$ for the differentiation of LN with ENE from LN without ENE showed an area under the ROC curve (AUC) value of $0.913 \pm 0.28(p<0.001$, Figure 2). At the best discriminative $\mathrm{SUV}_{\max }$ cut-off of 3.0, the sensitivity and specificity were $81.1 \%$ and $94.3 \%$, respectively (Youden index $=0.755$, Tables I and II).

\section{Discussion}

ENE is one of the most important factors for clinical decision making in radiotherapy planning in $\operatorname{HNSCC}(3,4)$. However, there is currently no established method for the diagnosis of ENE. The previously reported respective sensitivities and specificities are $85 \%$ and $88 \%$ with SUV cut-off of 2.25 for the oral cavity (AUC value of 0.864 ), $74 \%$ and $71 \%$ with SUV cut-off of 3.85 for the oropharynx (AUC value of 0.814 ), $80 \%$ and $74 \%$ with SUV cut-off of 2.65 for the hypopharynx (AUC value of 0.857 ), and $86 \%$ and $86 \%$ with SUV cut-off of 2.8 for the larynx (AUC value of 0.923) (7-10). Our results using the SUV cut-off method were similar with the high AUC value and Youden index. One possible reason is that we evaluated the diagnostic performance of FDG-PET/CT imaging with an adequate 6-week interval between imaging and surgery (11). Furthermore, our results suggested that FDG-PET using the single SUV cut-off method provides appropriate diagnostic value for identifying ENE in patients with HNSCC regardless of the primary subsite. Because HNSCC often involves more than one head and neck subsite, it may be reasonable to utilize the single SUV cut-off method regardless of the primary subsite.

Radiological evaluation using CT and MRI are commonly performed for the diagnosis of ENE. Steinkamp et al. (13) evaluated the diagnostic performance of CT for ENE in 165 patients with HNSCC. The sensitivity, specificity, positive predictive value, negative predictive value, and accuracy were $80.9 \%, 72.7 \%, 69.4 \%, 83.3 \%$, and $76.3 \%$, respectively. Prabhu et al. (14) evaluated diagnostic performance of CT for ENE in 432 patients with oral cavity or locally advanced/nonfunctional laryngeal cancer. The sensitivity, specificity, positive predictive value, negative predictive value, and accuracy were $43.7 \%, 97.7 \%, 82.6 \%, 87.3 \%$, and $86.8 \%$, respectively. Steinkamp et al. (15) evaluated diagnostic performance of MRI for ENE in 110 patients with HNSCC. The sensitivity, specificity, positive predictive value, negative predictive value, and accuracy were $74.4 \%$, $72.2 \%, 76.1 \%, 70.3 \%$, and $73.4 \%$, respectively. Another 


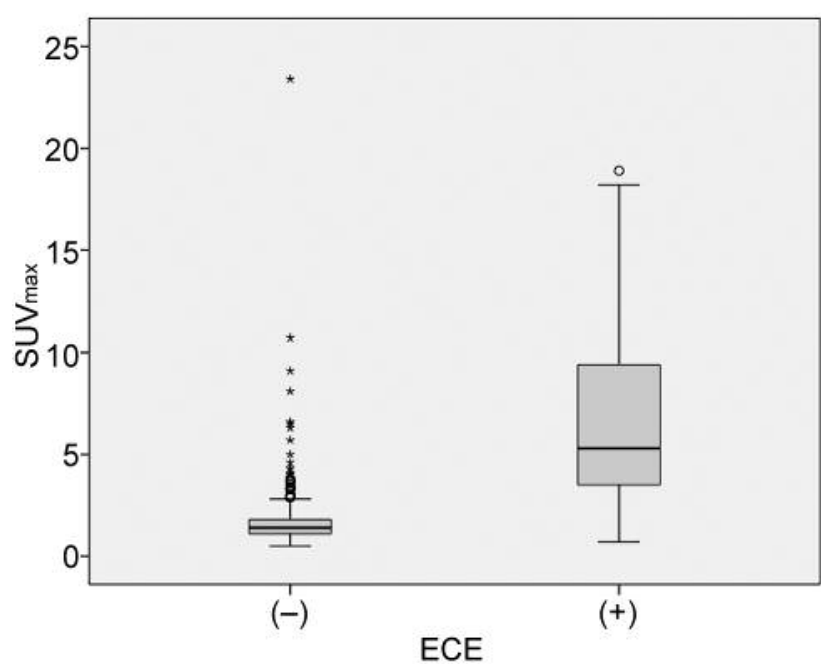

Figure 1. Relationship between maximum standardized uptake value and lymph nodes with and without extranodal extension.

Table I. Comparison between diagnosis of extranodal extension on FDG-PET/CT with SUV $V_{\max }$ cut-off of 3.0 and pathological results.

\begin{tabular}{llrr}
\hline & \multicolumn{2}{c}{ ENE on pathology } & \\
\cline { 2 - 3 } ENE on FDG-PET/CT & \multicolumn{1}{c}{ Yes } & No & Total \\
\hline Yes & 43 & 29 & 72 \\
No & 10 & 484 & 494 \\
Total & 53 & 513 & 566 \\
\hline
\end{tabular}

ENE: Extranodal extension.

Table II. Diagnostic accuracy of FDG-PET/CT with $S U V_{\max }$ cut-off of 3.0 for extranodal extension.

\begin{tabular}{ll}
\hline & $\%(95 \% \mathrm{CI})$ \\
\hline Sensitivity & $81.1(70.5-88.8)$ \\
Specificity & $94.3(93.3-95.1)$ \\
PPV & $59.7(51.9-65.4)$ \\
NPV & $98.0(96.8-98.8)$ \\
Accuracy & $93.1(91.1-94.5)$ \\
\hline
\end{tabular}

PPV: Positive predictive value; NPV: negative predictive value.

group (16) compared the diagnostic performance of MRI with that of CT for ENE in 17 patients with HNSCC. The sensitivity, specificity, and accuracy were $65 \%, 93 \%$, and $73 \%$ for $\mathrm{CT}$, and $78 \%, 86 \%$, and $80 \%$ for MRI, respectively. They found no significant difference between the two

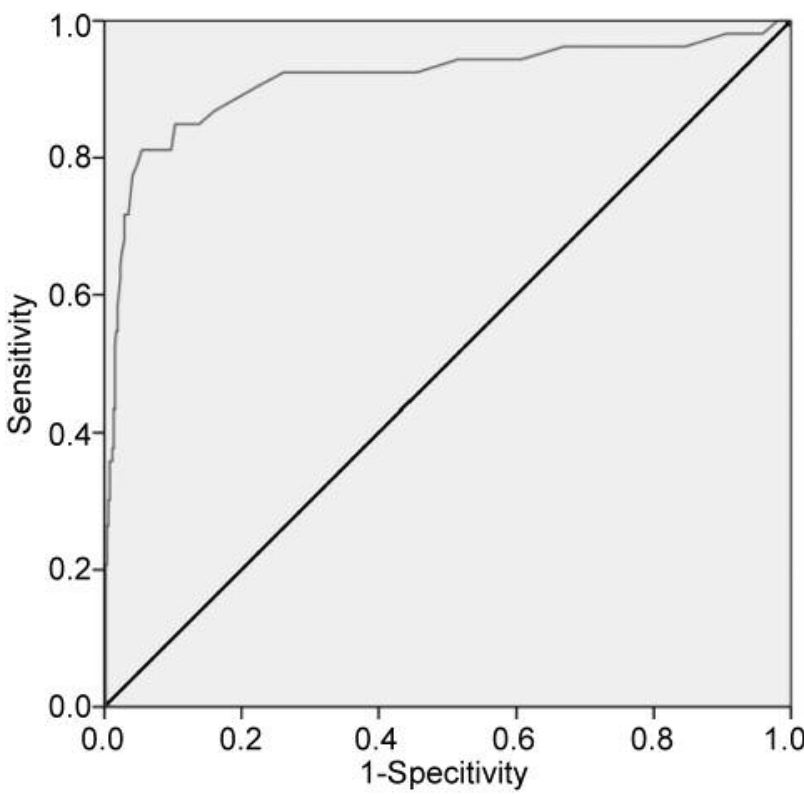

Figure 2. Receiver operating characteristics curve of maximum standardized uptake value for differentiating lymph nodes with and without extranodal extension.

modalities for either sensitivity $(p=0.1317)$ or specificity $(p=0.3137)$. Our results suggested that diagnostic performance of FDG-PET/CT imaging using the SUV cutoff method was comparable. The commonly-used diagnostic criteria for ENE on CT and MRI are the presence of an indistinct nodal margin, irregular nodal capsular enhancement, or infiltration into the adjacent fat or muscle $(16,17)$. However, there is a wide interobserver variation in the interpretation of these criteria. King et al. (16) reported that interobserver variation for the detection of ENE based on CT and MRI showed a kappa coefficient of 0.6077 and 0.4966 , respectively. The diagnostic performance of the nodal size criteria, which is a more objective method than these radiological features, is not sufficient for the diagnosis of ENE. Previous studies suggested that ENE exists in more than $40 \%$ of LNs with a size of $<10 \mathrm{~mm}(18,19)$. Zoumalan et al. (20) evaluated the relationship between nodal size and ENE based on CT imaging and ND specimens of 17 patients. LN diameter was not significantly different between LNs with ENE and those without ( $p=0.83$ ). We recommend the SUV cut-off method as an objective and simplified method to identify ENE in patients with HNSCC, regardless of subsite. The CTV should be defined to cover microscopic spread around LNs with high SUV $\mathrm{max}_{\max }$.

There are some limitations to our study. First, it is retrospective in nature, therefore the potential for selection bias exists, as preoperative imaging may have influenced the decision to perform surgery. Second, we determined an 
$\mathrm{SUV}_{\max }$ of 3.0 as the optimal cut-off to identify ENE. However, this cut-off value was based on PET/CT imaging at a single institution. Previous studies have suggested that SUV may change between individual PET/CT scanners, acquisition mode, and image reconstruction and processing parameters (21). Therefore, optimal cut-off value may be slightly different among each institution's PET/CT imaging protocol.

In conclusion, FDG-PET findings using a SUV $\mathrm{max}_{\text {max }}$ cut-off of 3.0 provides appropriate diagnostic value in identifying ENE in patients with HNSCC regardless of the primary subsite. The CTV should be defined to cover microscopic spread around LNs with $\mathrm{SUV}_{\max }$ above this value due to their greater risk of harbouring unknown ENE.

\section{Conflicts of Interest}

The Authors declare that they have no conflicts of interest related to this study.

\section{Authors' Contributions}

RT developed the study design, collected, analyzed, and interpreted data, performed statistical analysis, and wrote the manuscript. TS, TM, SS, and TW developed the study design, analyzed and interpreted data. YK analyzed data and performed statistical analysis. DM, RY, FS, and NT collected data. HA, YO, HN, and NO developed the study design and interpreted data. All Authors have read and approved the final manuscript.

\section{Acknowledgements}

This work was supported by the JSPS KAKENHI (grant number 18K07758).

\section{References}

1 Gregoire V, Grau C, Lapeyre M and Maingon P: Target volume selection and delineation $(\mathrm{t}$ and $\mathrm{n})$ for primary radiation treatment of oral cavity, oropharyngeal, hypopharyngeal and laryngeal squamous cell carcinoma. Oral Oncol 87: 131-137, 2018. PMID: 30527228. DOI: 10.1016/j.oraloncology.2018.10.034

2 Johnson JT, Myers EN, Bedetti CD, Barnes EL, Schramm VL Jr. and Thearle PB: Cervical lymph node metastases. Incidence and implications of extracapsular carcinoma. Arch Otolaryngol 111(8): 534-537, 1985. PMID: 4026664. DOI: 10.1001/ archotol.1985.00800100082012

3 Mermod M, Tolstonog G, Simon C and Monnier Y: Extracapsular spread in head and neck squamous cell carcinoma: A systematic review and meta-analysis. Oral Oncol 62: 60-71, 2016. PMID: 27865373. DOI: $10.1016 /$ j.oraloncology.2016.10.003

4 Apisarnthanarax S, Elliott DD, El-Naggar AK, Asper JA, Blanco A, Ang KK, Garden AS, Morrison WH, Rosenthal D, Weber RS and Chao KS: Determining optimal clinical target volume margins in head-and-neck cancer based on microscopic extracapsular extension of metastatic neck nodes. Int J Radiat Oncol Biol Phys 64(3): 678-683, 2006. PMID: 16243444. DOI: 10.1016/j.ijrobp.2005.08.020
5 Murakami R, Uozumi H, Hirai T, Nishimura R, Shiraishi S, Ota K, Murakami D, Tomiguchi S, Oya N, Katsuragawa S and Yamashita Y: Impact of fdg-pet/ct imaging on nodal staging for head-and-neck squamous cell carcinoma. Int J Radiat Oncol Biol Phys 68(2): 377382, 2007. PMID: 17321066. DOI: 10.1016/j.ijrobp.2006.12.032

6 Wright CL, Washington IR, Bhatt AD and Knopp MV: Emerging opportunities for digital pet/ct to advance locoregional therapy in head and neck cancer. Semin Radiat Oncol 29(2): 93-101, 2019. PMID: 30827458. DOI: 10.1016/j.semradonc.2018.11.005

7 Joo YH, Yoo IR, Cho KJ, Park JO, Nam IC and Kim MS: Extracapsular spread and fdg pet/ct correlations in oral squamous cell carcinoma. Int J Oral Maxillofac Surg 42(2): 158163, 2013. PMID: 23219708. DOI: 10.1016/j.ijom.2012.11.006

8 Joo YH, Yoo Ie R, Cho KJ, Park JO, Nam IC, Kim CS and Kim MS: Relationship between extracapsular spread and fdg pet/ct in oropharyngeal squamous cell carcinoma. Acta Otolaryngol 133(10): 1073-1079, 2013. PMID: 24032571. DOI: $10.3109 /$ 00016489.2013 .799292

9 Joo YH, Yoo Ie R, Cho KJ, Park JO, Nam IC and Kim MS: Extracapsular spread in hypopharyngeal squamous cell carcinoma: Diagnostic value of FDG PET/CT. Head Neck 35(12): 1771-1776, 2013. PMID: 23508670. DOI: 10.1002/hed.23239

10 Chun BJ, Yoo Ie R, Joo YH, Nam IC, Cho JH, Kim CS, Cho KJ and Kim MS: Efficacy of 18f-fluorodeoxyglucose positron emission tomography/ct imaging for extracapsular spread of laryngeal squamous cell carcinoma. Head Neck 38(2): 290-293, 2016. DOI: $10.1002 /$ hed 23889

11 Geltzeiler M, Clayburgh D, Gleysteen J, Gross ND, Hamilton B, Andersen $\mathrm{P}$ and Brickman D: Predictors of extracapsular extension in hpv-associated oropharyngeal cancer treated surgically. Oral Oncol 65: 89-93, 2017. PMID: 28109475. DOI: 10.1016/j.oraloncology.2016.12.025

12 Toya R, Matsuyama T, Saito T, Imuta M, Shiraishi S, Fukugawa Y, Iyama A, Watakabe T, Sakamoto F, Tsuda N, Shimohigashi Y, Kai Y, Murakami R, Yamashita Y and Oya N: Impact of hybrid fdg-pet/ct on gross tumor volume definition of cervical esophageal cancer: Reducing interobserver variation. J Radiat Res 60(3): 348352, 2019. PMID: 30864652. DOI: 10.1093/jrr/rrz004

13 Steinkamp HJ, van der Hoeck E, Bock JC and Felix R: [the extracapsular spread of cervical lymph node metastases: The diagnostic value of computed tomography]. Rofo 170(5): 457462, 1999. PMID: 10370409. DOI: 10.1055/s-2007-1011073

14 Prabhu RS, Magliocca KR, Hanasoge S, Aiken AH, Hudgins PA, Hall WA, Chen SA, Eaton BR, Higgins KA, Saba NF and Beitler JJ: Accuracy of computed tomography for predicting pathologic nodal extracapsular extension in patients with headand-neck cancer undergoing initial surgical resection. Int $\mathrm{J}$ Radiat Oncol Biol Phys 88(1): 122-129, 2014. PMID: 24331658. DOI: $10.1016 /$ j.ijrobp.2013.10.002

15 Steinkamp HJ, Beck A, Werk M and Felix R: Extracapsular spread of cervical lymph node metastases: Diagnostic value of magnetic resonance imaging. Rofo 174(1): 50-55, 2002. PMID: 11793284. DOI: $10.1055 / \mathrm{s}-2002-19533$

16 King AD, Tse GM, Yuen EH, To EW, Vlantis AC, Zee B, Chan $\mathrm{AB}$, van Hasselt AC and Ahuja AT: Comparison of ct and mr imaging for the detection of extranodal neoplastic spread in metastatic neck nodes. Eur J Radiol 52(3): 264-270, 2004. PMID: 15544904. DOI: 10.1016/j.ejrad.2004.03.004

17 Souter MA, Allison RS, Clarkson JH, Cowan IA, Coates MH and Wells JE: Sensitivity and specificity of computed tomography for 
detection of extranodal spread from metastatic head and neck squamous cell carcinoma. J Laryngol Otol 123(7): 778-782, 2009. PMID: 19134244. DOI: 10.1017/S0022215109004332

18 Hirabayashi H, Koshii K, Uno K, Ohgaki H, Nakasone Y, Fujisawa T, Syouno N, Hinohara T and Hirabayashi K: Extracapsular spread of squamous cell carcinoma in neck lymph nodes: Prognostic factor of laryngeal cancer. Laryngoscope 101(5): 502-506, 1991. PMID: 2030629. DOI: 10.1288/00005537-199105000-00010

19 Ghadjar P, Schreiber-Facklam H, Grater R, Evers C, Simcock M, Geretschlager A, Blumstein NM, Zbaren P, Zimmer Y, Wilkens L and Aebersold DM: Quantitative analysis of extracapsular extension of metastatic lymph nodes and its significance in radiotherapy planning in head and neck squamous cell carcinoma Int J Radiat Oncol Biol Phys 76(4): 1127-1132, 2010. PMID: 19647955. DOI: 10.1016/j.jijrobp.2009.03.065
20 Zoumalan RA, Kleinberger AJ, Morris LG, Ranade A, Yee H, DeLacure MD and Myssiorek D: Lymph node central necrosis on computed tomography as predictor of extracapsular spread in metastatic head and neck squamous cell carcinoma: Pilot study. J Laryngol Otol 124(12): 1284-1288, 2010. PMID: 20573293. DOI: $10.1017 / \mathrm{S} 0022215110001453$

21 Adams MC, Turkington TG, Wilson JM and Wong TZ: A systematic review of the factors affecting accuracy of suv measurements. AJR Am J Roentgenol 195(2): 310-320, 2010. PMID: 20651185. DOI: 10.2214/AJR.10.4923

Received February 9, 2020

Revised February 15, 2020

Accepted February 19, 2020 